\title{
A Regularity Lemma for Functions of Several Variables
}

J. L. Journé

Dedicated to the memory of José Luis Rubio de Francia

We shall prove the following

Theorem. Let $F_{s}$ and $F_{u}$ be two continuous transverse foliations with uniformly smooth leaves, of some manifold. If $f$ is uniformly smooth along the leaves of $F_{s}$ and $F_{u}$, then $f$ is smooth.

In the case where the foliations are the stable and unstable foliations of an Anosov diffeomorphism, this theorem is proved in [1] using elliptic theory and in [2] using Fourier transform. Both proofs make use of the fact that the foliations are absolutely continuous. For the applications of [3] one does not even have foliations: only two transverse submanifolds through each point with continuity of the tangent spaces, and $f$ is assumed smooth along these submanifolds. Our proof is the only one which can handle these assumptions and moreover, is totally elementary.

In order to avoid notational complications we present the proof in dimension 2 . The modifications needed in higher dimensions are very minor and will be omitted.

Let $n \in \mathbb{N}$ and $\alpha \in] 0,1\left[\right.$. We shall prove the $C^{n, \alpha}$ version of the theorem, which is sufficient, and this will show that there is no loss in regularity. The problem being local we may work in some open set $U \subseteq \mathbb{R}^{2}$. Let $f$ be uniformly $C^{n, \alpha}$ along the leaves of $F_{s}$ and $F_{u}$. To show that $f$ is $C^{n, \alpha}$, by a lemma of Campanato [4], it is enough to show that at each point $M$ in $U$ we can find a $C^{n, \alpha}$ function $f_{M}$ such that $\left|f_{M}(P)-f(P)\right| \leqslant O\left(\|P M\|^{n+\alpha}\right)$ for all $P$ in $U$, 
all constants being locally uniform. After a $C^{n, \alpha}$ change of variables we can assume that the leaves $L_{u}$ and $L_{s}$ passing through $M$ are the coordinate axes, and that $f$ vanishes on these two leaves by replacing $f(x, y)$ by $f(x, y)-f(x, 0)$ $-f(0, y)+f(0,0)$. In these new coordinates we shall look for $f_{M}$ to be a polynomial of total degree $n$. Then it will have to be the Taylor polynomial of order $n$ of $f$ at $M$. This polynomial will be obtained as the limit of polynomials of degree $n$ in each variable, determined by interpolating $f$ on sets of points converging to $M$.

For $r<1$, let $x_{k}=y_{k}=r^{k}$ for $k \in \mathbb{N}$. For every point $P$, let $L^{u}(P)$ and $L^{s}(P)$ be the two leaves of $F_{s}$ and $F_{u}$ containing $P$. Let $M_{k, l}$ be $L^{u}\left(\left(x_{k}, 0\right)\right) \cap L^{s}\left(\left(0, y_{l}\right)\right)$. Let $S(k, l)=\{(0,0)\} \cup\left\{\left(x_{k^{\prime}}, 0\right): k \leqslant k^{\prime}<k+n\right\} \cup\left\{\left(0, y_{l^{\prime}}\right): l \leqslant l^{\prime}<l+n\right\} \cup$ $\left\{\left(M_{k, l}\right): k \leqslant k^{\prime}<k+n, l \leqslant l^{\prime}<l+n\right\}$. We want for $k$ and $l$ large enough, with $l=k$ or $k+1$, a polynomial of degree $n$ in $x$ and in $y$ which interpolates $f$ on $S(k, l)$. In one variable, in order to interpolate a function $g$ by a polynomial of degree $n$ one needs $(n+1)$ distinct reals $z_{0}, \ldots, z_{n}$. If $R$ denotes $\sup _{0 \leq i \leq n}\left|z_{i}\right|$ and $\eta$ denotes inf $i \neq l\left|z_{i}-z_{l}\right|$, and $c_{p}, 0 \leqslant p \leqslant n$ are the coefficients of the interpolating polynomial, then

$$
\sum_{0 \leq p \leq n}\left|c_{p}\right| R^{p} \leqslant C \sup _{0 \leq i \leq n}\left|g\left(z_{i}\right)\right|,
$$

where $C$ remains bounded if $R / \eta$ remains bounded. In dimension 2 one can interpolate a function by a polynomial of degree $n$ in each variable on the cartesian product of two sets of $(n+1)$ real numbers. However, we will need to interpolate on sets of the type $S(k, l)$, which are not products, but close enough, because the foliations are continuous. The following lemma, which is probably well known, will allow us to do so.

Lemma 1. For each $B>1$, there exist $\epsilon>0$ and $C>0$ with the following properties. There exists $\epsilon>0$ with the following property. Let $\left(z_{k, l}\right)_{0 \leq k \leq n, 0 \leq l \leq n}$ be a collection of points in $\mathbb{R}^{2}$ for which there exists $2 n+2$ real numbers $\left(x_{k}\right)_{0 \leq k \leq n}$ and $\left(y_{l}\right)_{0 \leq l \leq n}$, such that

$$
\left|z_{k, l}-\left(x_{k}, y_{l}\right)\right| \leqslant \epsilon \eta,
$$

where

$$
\eta=\inf _{(k, l) \neq\left(k^{\prime}, l^{\prime}\right)}\left|z_{k, l}-z_{k^{\prime}, l^{\prime}}\right| .
$$

Let

$$
R=\sup _{k, l}\left|z_{k, l}\right|
$$

and suppose $R / \eta<k$. 
Then given $(n+1)^{2}$ real numbers $\left(b_{k, l}\right)_{0 \leq k \leq n, 0 \leq l \leq n}$, there exists a unique polynomial

$$
p(x, y)=\sum_{\substack{0 \leq p \leq n \\ 0 \leq q \leq n}} c_{p q} x^{p} y^{q}
$$

such that $p\left(z_{k, l}\right)=b_{k, l}$. Also

$$
\sum_{p, q}\left|c_{p, q}\right| R^{p+q} \leqslant C \sup _{(k, l)}\left|b_{k, l}\right| .
$$

Proof. We fix $B>1$. By iterating one-dimensional interpolation one can construct a polynomial $P_{1}$, of degree $n$ in $x$ and in $y$, such that $P_{1}\left(x_{k}, y_{l}\right)=b_{k, l}$ for $0 \leqslant k, l \leqslant n$. Then it is easy to see that for $\epsilon>0$ small enough

$$
\sup _{k, l}\left|b_{k, l}-P_{1}\left(z_{k, l}\right)\right| \leqslant \frac{1}{2} \sup _{k, l}\left|b_{k, l}\right|
$$

Then one replaces $b_{k, l}$ by $b_{k, l}-P_{1}\left(z_{k, l}\right)$ and constructs similarly $P_{2}$. Then

$$
\begin{aligned}
\sup _{k, l}\left|b_{k, l}-P_{1}\left(z_{k, l}\right)-P_{2}\left(z_{z, l}\right)\right| & \leqslant \frac{1}{2} \sup _{k, l}\left|b_{k, l}-P_{1}\left(z_{k, l}\right)\right| \\
& \leqslant \frac{1}{4} \sup _{k, l}\left|b_{k, l}\right| .
\end{aligned}
$$

It is clear that repeating the process will yield a convergent series whose sum will be $P$. The inequality (2) will follow from (1) and uniqueness follows from (2). We omit the details.

The continuity of the foliations implies that $\left|M_{k, l}-\left(x_{k}, y_{l}\right)\right|=O\left(\left|\left(x_{k}, y_{l}\right)\right|\right)$. It follows that for $k_{0}$ large enough and $k \geqslant k_{0}, S(k, k)$ and $S(k, k+1)$ satisfy uniformly the assumptions of the lemma. Let $k \geqslant k_{0}$ be fixed and $P$ be the polynomial of degree $n$ in $x$ and in $y$ which interpolates $f$ on $S(k, k)$ and let $P^{\prime}$ be the same for $S(k, k+1)$ and let $c_{p, q}$ and $c_{p, q}^{\prime}$ denote their coefficients.

We want to show

$$
\left|c_{p, q}^{\prime}-c_{p, q}\right|=O\left(r^{k(n+\alpha-p-q)}\right) .
$$

By Lemma 1 it is enough to show that if $(x, y) \in S_{k, k+1}$

$$
\left|P^{\prime}(x, y)-P(x, y)\right|=O\left(r^{k(n+\alpha)}\right) .
$$

Observe that $P$ and $P^{\prime}$ agree on $S_{k, k+1}$, except on the $n$ points $M_{k^{\prime}, k+n}$, $k \leqslant k^{\prime}<k+n$. On these points $P^{\prime}$ agrees with $f$. Hence we just need to estimate $f-P$ at those $n$ points. Now we fix $k^{\prime}$ and look at $L^{u}\left(\left(x_{k^{\prime}}, 0\right)\right)$, 
which we parametrize by the $y$-coordinate. A point on $L^{u}\left(\left(x_{k^{\prime}}, 0\right)\right)$ is denoted $\left(x_{k^{\prime}}(y), y\right)=M_{y}$. Since $f$ and $P$ are both $C^{n, \alpha}$ along $L^{u}\left(\left(x_{k^{\prime}}, 0\right)\right)$, and coincide on $(n+1)$ points whose minimum and maximum distance are of the order of $r^{k}$, it follows that if $|y| \leqslant C r^{k}$

$$
\left|(f-P)\left(M_{y}\right)\right| \leqslant C r^{k(n+\alpha)}\left\|\frac{d^{n}}{d y^{n}}(f-P)\right\|_{\alpha} .
$$

By the assumption on $f$,

$$
\left\|\frac{d^{n}}{d y^{n}} f\right\|_{\alpha} \leqslant c
$$

So we must estimate

$$
\left\|\frac{d^{n}}{d y^{n}} P\left(x_{k^{\prime}}(\bullet), \bullet\right)\right\|_{\alpha},
$$

where we are only interested in values of $y$ such that $|y| \leqslant C r^{k}$. If $x_{k^{\prime}}(y)$ was constant, this would be zero. The foliations being continuous, $x_{k^{\prime}}$ is close to be a constant, in that for any $\delta>0$ and for $k$ large enough $\left|x_{k^{\prime}}^{\prime}(y)\right|<\delta$ if $|y| \leqslant C r^{k}$.

Lemma 2. Let $\delta>0$. Then for $k$ large enough

$$
\left\|\frac{d^{n}}{d y^{n}} x_{k}^{p}(y) y^{q}\right\|_{C^{\alpha}\left[-C r^{k}, C r^{k}\right]} \leqslant C \delta\left(r^{k}\right)^{p+q-n-\alpha}
$$

if $p$ and $q$ are non-zero and $p+q>n$.

This follows trivially from Leibniz' rule. Also one has to use that $x_{k}$ is $C^{n, \alpha}$ and that if $y$ and $y^{\prime}$ lie in $\left[-C r^{k}, C r^{k}\right]$, then

$$
\sup _{y \neq y^{\prime}} \frac{\left|x_{k^{\prime}}(y)-x_{k^{\prime}}\left(y^{\prime}\right)\right|}{\left|y-y^{\prime}\right|^{\alpha}} \leqslant \delta C\left(r^{k}\right)^{1-\alpha} .
$$

We omit the details.

Notice that because we have set $f$ to be zero on the $x$ and $y$ axes, no term of the form $c_{p, 0} x^{p}$ or $c_{0, q} y^{q}$ appears in $P$. In the case where $p+q \leqslant n$, instead of (5) we use

$$
\left\|\frac{d^{n}}{d y^{n}} x_{k}^{p}(y) y^{q}\right\|_{C^{\alpha}} \leqslant C
$$


From (4), (5) and (6) we obtain, if $|y| \leqslant C r^{k}$

$$
\left|\left(P^{\prime}-P\right)\left(M_{k, k^{\prime}}\right)\right| \leqslant C r^{k(n+\alpha)}+\delta \sum_{p+q>n}\left|c_{p q}\right| r^{k(p+q)}+\sum_{p+q \leq n}\left|c_{p q}\right| r^{k(n+\alpha)} .
$$

By Lemma 1, this implies

(7) $\sum\left|c_{p, q}^{\prime}-c_{p, q}\right|\left(r^{k}\right)^{p+q}$

$$
\leqslant C\left\{r^{k(n+\alpha)}+\delta \sum_{p+q>n}\left|c_{p, q}\right| r^{k(p+q)}+\sum_{p+q \leq n}\left|c_{p, q}\right| r^{k(n+\alpha)}\right\} .
$$

We denote by $P_{2 k}$ for all $k$ the interpolation polynomial corresponding to $S(k, k)$ and $P_{2 k+1}$ the one corresponding to $S(k, k+1)$. Then (7) relates the coefficients of $P_{2 k+1}$ to those of $P_{2 k}$. The same proof shows that the coefficients of $P_{2 k+2}$ are related to those of $P_{2 k+1}$ in the same way. Then, we want to show by induction on $m$, that if $c_{p, q}$ is the coefficient of $x^{p} y^{q}$ in $P_{m}$, and $m>m_{0}$,

$$
\left|c_{p, q}\right| \leqslant K \sum_{j=m_{0}}^{m-1}\left(r^{j / 2}\right)^{n+\alpha-p-q},
$$

for $K$ and $m_{0}$ large enough.

Assume that this is true. Then it will be true for $m+1$, by (7), if

(9) $C\left\{r^{k(n+\alpha)}+\delta K \sum_{\substack{p+q>n \\ m_{0} \leq j \leq m-1}} r^{k(p+q)}\left(r^{j / 2}\right)^{n+\alpha-p-q}\right.$

$$
\left.+K \sum_{\substack{p+q \leq n \\ m_{0} \leq j \leq m-1}}\left(r^{j / 2}\right)^{n+\alpha-p-q} r^{k(n+\alpha)}\right\} \leqslant K r^{m(n+\alpha) / 2}
$$

where $k=[m / 2]$.

The second term is less than $C \delta K r^{m(n+\alpha) / 2}$ and the third one is less than $C K r^{(n+\alpha)\left(m+m_{0}\right) / 2}$. So by choosing $m_{0}$ large enough, $\delta$ small enough, and $K$ large enough so that (8) is satisfied for $m=m_{0}+1$, it follows by induction that (8) is satisfied for all $m>m_{0}$. Then the same is true for (9), (7) and (3).

We are going to see that this implies the existence of a polynomial $Q$ of degree $n$, independent of $m$, such that

$$
\left|Q-P_{m}\right| \leqslant C r^{m(n+\alpha) / 2} \text { for }|x|,|y| \leqslant C r^{m / 2} .
$$

The coefficients $l_{p, q}$ of $Q$ are obtained in the following way. If $p+q>n$, let $l_{p, q}=0$. If $p+q \leqslant n$ let

$$
l_{p, q}=\lim _{m \rightarrow+\infty} c_{p, q} .
$$


From (3) we know that if $p+q>n,\left|c_{p, q}\right|=O\left(r^{m(n+\alpha-p-q) / 2}\right)$ and if $p+$ $q \leqslant n,\left|c_{p, q}-l_{p, q}\right|=O\left(r^{m(n+\alpha-p-q) / 2}\right)$. This implies (10).

From (3), (4), (5) and (6), we know that

$$
\left|\left(f-P_{m}\right)\left(M_{y}\right)\right| \leqslant C r^{m(n+\alpha) / 2} \quad \text { if }|y| \leqslant C r^{k} .
$$

Using the triangle inequality and (10) we see that

$$
\left|(Q-f)\left(x_{k}(y), y\right)\right| \leqslant C r^{m(n+\alpha) / 2} \quad \text { if } \quad|y| \leqslant C r^{k} .
$$

Therefore if $|y| \leqslant 2|x|$ and $(x, y) \in \lim \bigcup_{k>0} L^{u}\left(r^{k}, 0\right)$ then:

$$
|Q(x, y)-f(x, y)| \leqslant C|(x, y)|^{n+\alpha} .
$$

Let $\epsilon>0$ be a fixed small number. If we replace in the preceding statement the sequence $r^{k}$ by another sequence $\alpha_{k}$ such that $r^{k+1} \leqslant\left|\alpha_{k}\right| \leqslant r^{k-1}$ for all $k$ and $\left|\alpha_{k}-\alpha_{k-1}\right| \geqslant \epsilon r^{k}$ then one obtains the same conclusion with a possibly different polynomial $\tilde{Q}$. However by mixing two such sequences one sees that the polynomial $\tilde{Q}$ is actually independent of the sequence and that (11) holds with the only assumption $|y|<2|x|$. Also (11) holds with a possibly different polynomial $Q^{\prime}$ of degree $n$ if $|x|<2|y|$. But then $\left|\left(Q-Q^{\prime}\right)(x, y)\right|=O\left(|(x, y)|^{n+\alpha}\right)$ if $|y| / 2<|x|<2|y|$, so that $Q=Q^{\prime}$. Therefore (11) holds with no extra assumption on $(x, y)$. According to the lemma of Campanato already mentioned, this proves the theorem.

Remark. It is easy to see that if one of the two foliations is locally trivial, -that is, the leaves are, in some coordinates neighborhood, open subsets of parallel affine subspaces - then one can remove the hypothesis of continuity on the other foliation. Indeed if it is merely measurable, one can make its local oscillation arbitrarily small by a rescaling along the direction of the trivial foliation and this is enough to carry out the proof. It is to be noted that in this slightly different context the analytic version of the theorem is false. The counterexample suggests that analytic versions of such results require much more transverse regularity than their smooth counterparts. For example in dimension 2, one can show the analytic version of the theorem if the foliations have the following property: suppose that, in some coordinates, the leaves are the graphs of functions $\left.f_{y}, y \in\right]-R, R$ [, with $f_{y}(0)=y$. Then it is enough to assume that $y \rightarrow f_{y}$, which maps ] $-R, R$ [ into $H^{\infty}(\Omega)$, for some $\Omega$ open set of $\mathbb{C}$ containing the origin, is a lipschitzian function.

\section{Acknowledgement}

The author wishes to thank Rafael de la Llave and the referee for several comments which improved the exposition. 


\section{References}

[1] Llave, R. de la, Marco, J. M. y Moriyón, R. Canonical perturbation theory of Anosov systems and regularity results for the Livsic cohomology equation, Ann. of Math. 123(1986), 537-611.

[2] Hurder, S. y Katok, A. Differentiability, Rigidity and Godbillon-Vey Classes for Anosov Flows. To appear in Pub. Mat. I.H.E.S.

[3] Llave, R. de la. New invariant manifold theorems and applications, Preprint, 1988.

[4] Campanato, S. Proprietá di una famiglia di spazi funzionali. Ann. Sc. Norm. Super. Pisa, 18(1969), 137-160.

J. L. Journé

Princeton University

Mathematics Department

Princeton NJ 08544

U.S.A. 